\title{
Hydroxychloroquine in Patients with Rheumatic Disease Complicated by COVID-19: Clarifying Target Exposures and the Need for Clinical Trials
}

\author{
Stephen J. Balevic (D), Christoph P. Hornik (D), Thomas P. Green, Megan E.B. Clowse (D), \\ Daniel Gonzalez $\mathbb{D}$, Anil R. Maharaj, Laura E. Schanberg, Amanda M. Eudy $\mathbb{D}$, \\ Geeta K. Swamy (i), Brenna L. Hughes, and Michael Cohen-Wolkowiez (i)
}

ABSTRACT. Objective. To characterize hydroxychloroquine (HCQ) exposure in patients with rheumatic disease receiving longterm HCQ compared to target concentrations with reported antiviral activity against the coronavirus disease 2019 caused by SARS-CoV-2 (COVID-19).

Method. We evaluated total HCQ concentrations in serum and plasma from published literature values, frozen serum samples from a pediatric systemic lupus erythematosus trial, and simulated concentrations using a published pharmacokinetic model during pregnancy. For each source, we compared observed or predicted HCQ concentrations to target concentrations with reported antiviral activity against SARS-CoV-2.

Results. The average total serum/plasma HCQ concentrations were below the lowest SARS-CoV-2 target of $0.48 \mathrm{mg} / \mathrm{l}$ in all studies. Assuming the highest antiviral target exposure (total plasma concentration of $4.1 \mathrm{mg} / \mathrm{l}$ ), all studies had about one-tenth the necessary concentration for in vitro viral inhibition. Pharmacokinetic model simulations confirmed that pregnant adults receiving common dosing for rheumatic diseases did not achieve target exposures; however, the models predict that a dosage of $600 \mathrm{mg}$ once a day during pregnancy would obtain the lowest median target exposure for most patients after the first dose.

Conclusion. We found that the average patient receiving treatment with HCQ for rheumatic diseases, including children and non-pregnant/pregnant adults, are unlikely to achieve total serum or plasma concentrations shown to inhibit SARS-CoV-2 in vitro. Nevertheless, patients receiving HCQ long term may have tissue concentrations far exceeding that of serum/plasma. Because the therapeutic window for HCQ in the setting of SARS-CoV-2 is unknown, well-designed clinical trials that include patients with rheumatic disease are urgently needed to characterize the efficacy, safety, and target exposures for HCQ. (First Release June 15 2020; J Rheumatol 2020;47:1424-30; doi:10.3899/jrheum.200493)

Key Indexing Terms:

COVID-19 PREGNANCY

SYSTEMIC LUPUS ERYTHEMATOSUS

HYDROXYCHLOROQUINE RHEUMATIC DISEASE

\begin{abstract}
From the Department of Rheumatology and Immunology, Duke University School of Medicine, Durham, North Carolina, USA; Duke Clinical Research Institute, Durham, North Carolina, USA; Department of Pediatrics, and the Division of Maternal-Fetal Medicine, Department of Obstetrics \& Gynecology, Duke University School of Medicine, Durham, North Carolina, USA; Department of Pediatrics, Northwestern University, Feinberg School of Medicine, Evanston, Illinois, USA; Division of Pharmacotherapy and Experimental Therapeutics, UNC Eshelman School of Pharmacy, The University of North Carolina at Chapel Hill, Chapel Hill, North Carolina, USA.

This study was supported by the Rheumatology Research Foundation's Scientist Development Award, the Thrasher Research Fund, the Childhood Arthritis and Rheumatology Research Alliance/Arthritis Foundation, the Derfner Foundation, NIGMS/NICHD (2T32GM086330-06), NICHD (5R01-HD076676-04, HHSN275201000003I), and a Duke Health/Private Diagnostic Clinic ENABLE grant. The Atherosclerosis Prevention in Pediatric Lupus Erythematosus [APPLE (ClinicalTrials. gov: NCT00065806)] trial is supported by the US National Institutes of Health (NIH) National Institute of Arthritis and Musculoskeletal and Skin Diseases (NIAMS) contract NO1-AR-2-2265, the Edna and Fred L. Mandel Jr. Center for Hypertension and Atherosclerosis, and Pfizer, which provided atorvastatin and matching placebo.
\end{abstract}

S.J.B. receives support from the NIH (5R01-HD076676-04, 1R01HD083003-01, HHSN275201000003I, HHSN275201800003I, HHSN272201500006I 5U24-TR001608-03), the US Food and Drug Administration (5U18FD006298-03), the Patient-Centered Outcomes Research Institute (PCORI), the Rheumatology Research Foundation's Scientist Development Award, the Thrasher Research Fund, and the Childhood Arthritis and Rheumatology Research Alliance/Arthritis Foundation. C.P.H. receives salary support for research from the National Institute for Child Health and Human Development (NICHD, 1K23HD090239; R13HD102136), National Heart Lung and Blood Institute (R61/R33HL147833), FDA (1R01-FD006099, PI: Laughon; and 5U18-FD006298, PI: Benjamin), the US government for his work in pediatric clinical pharmacology (Government Contract HHSN275201800003I, PI: Benjamin under the Best Pharmaceuticals for Children Act), the nonprofit Burrhoughs Wellcome Fund, and other sponsors for drug development in adults and children (dcri.org/about-us) conflict-of-interest). D.G. receives support for research from the Eunice Kennedy Shriver NICHD (5R01HD096435). A.M. receives research support from the Thrasher Research Fund (www.thrasherresearch.org). L.E.S. receives support for research from the NIH (U19AR069522), PCORI (8177), and the Childhood Arthritis and Rheumatology Research Alliance. She is on the Data Safety Monitoring Board for investigational 
The coronavirus disease 2019 (COVID-19) pandemic caused by the novel coronavirus SARS-CoV-2 is causing substantial morbidity and mortality across the globe ${ }^{1,2}$. Because of preliminary data suggesting antiviral properties, hydroxychloroquine (HCQ) is being investigated as a potential treatment for SARS-CoV-2. Antimalarial drugs such as HCQ may inhibit viral replication by interfering with surface receptor binding, endosome-mediated cell entry, nucleic acid replication, and maturation of the viral protein ${ }^{3,4}$. Recently, these findings were supported by an in vitro study in which HCQ inhibited viral replication in SARS-CoV-2 infected Vero cells ${ }^{4}$. In the same publication, the authors used a physiologically based pharmacokinetic (PBPK) modeling approach to achieve target lung concentrations and suggested an HCQ loading dose in non-pregnant adults of $400 \mathrm{mg}$ every $12 \mathrm{~h}$ for 1 day, followed by a maintenance dose of $200 \mathrm{mg}$ every $12 \mathrm{~h}$ for 4 days. However, other PBPK modeling suggests acute dosing for HCQ may not achieve target unbound lung concentrations quickly ${ }^{5}$.

HCQ is a standard-of-care immune-modulating drug used to treat systemic lupus erythematosus (SLE) and other rheumatic diseases ${ }^{6}$. Owing to the time needed to reach steady state, patients receiving longterm HCQ eventually achieve higher blood and serum/plasma concentrations over time ${ }^{7}$. As a result, patients with rheumatic disease who are treated with HCQ long term may be more likely to achieve HCQ exposures that inhibit SARS-CoV-2 in vitro. Nevertheless, no studies have examined the role of established HCQ therapy in adults and children with rheumatic disease who are exposed to SARS-CoV-2, and it is unknown whether standard dosing strategies achieve target HCQ exposure. Therefore, the purpose of this study was to (1) compare HCQ serum/plasma concentrations observed in the clinical setting to those with purported antiviral activity, and (2) provide an example using population PK modeling to conduct dosing simulations, as well as demonstrate the utility of modeling to guide clinical trials using HCQ as a treatment for SARS-CoV-2 in patients with rheumatic disease (Study collaborators, Appendix 1).

\section{MATERIALS AND METHODS}

We conducted the study in accordance with the Declaration of Helsinki. Informed consent was obtained from all participants. The Duke Institutional Review Board reviewed and approved study protocols (Pro00000775, Pro00000756, Pro00103156).

Overall approach. We evaluated HCQ drug concentrations from 3 sources: (1) published literature values; (2) frozen serum samples from a completed pediatric SLE trial; and (3) simulated HCQ concentrations using a published PK model during pregnancy. For each of these sources, we compared observed or predicted HCQ concentrations to target HCQ concentrations with purported antiviral activity against SARS-CoV-2. First, we conducted a literature review and extracted summary statistics from published studies evaluating HCQ pharmacokinetics using serum or plasma in rheumatic diseases. Because the antiviral activity of HCQ is best characterized in serum and plasma ${ }^{4,8}$, we excluded studies describing HCQ concentrations in whole blood alone. Second, we analyzed HCQ concentrations in frozen serum samples from a biorepository of pediatric patients with SLE who completed a clinical trial ${ }^{9}$. Third, we used a published population PK model of pregnant women with rheumatic disease ${ }^{10}$ and compared total simulated serum concentrations to the concentration targets outlined below.

Target concentrations. We compared observed total plasma/serum concentrations from the clinical studies below with total target plasma concentrations of $0.48 \mathrm{mg} / \mathrm{l}$ and $4.1 \mathrm{mg} / \mathrm{l}$ proposed by Yao, et $a l^{4}$. The Yao targets are estimated to provide the total plasma concentrations equivalent to the in vitro half-maximal effective concentrations for SARS-CoV-2 treatment (EC50 of 0.72 and $6.14 \mathrm{uM}$ after $48 \mathrm{~h}$ and $24 \mathrm{~h}$, respectively), assuming an HCQ plasma protein binding of $50 \%{ }^{11}$. Although differences between serum and plasma HCQ concentrations have not been formally characterized, we expect the matrices to be highly similar ${ }^{12}$. Second, we compared results from our study to the average serum concentrations observed in the clinical study by Gautret, et al $(0.46 \mathrm{mg} / \mathrm{l} \pm 0.2)^{8}$.

Characteristics of included clinical studies: adult non-pregnancy studies. We included published data from 90 Japanese patients (270 plasma concentrations) with rheumatic disease enrolled in a clinical trial of $\mathrm{HCQ}^{13}$. Briefly, the average (range) characteristics of this population were 42.5 years (20-72) and a weight of $59.2 \mathrm{~kg}(38.6-103.7)$. Overall, $66(73.3 \%)$ were female and $50(55.6 \%)$ had SLE. We computed average plasma HCQ concentrations by taking the area under the curve divided by the dosing interval $(24 \mathrm{~h})$. We also included published data from 276 patients with SLE in a study from China ${ }^{14}$. The average (SD) characteristics of this cohort product trials for UCB (Cimzia) and Sanofi (sarilumab). Sanofi is a maker of hydroxychloroquine. Samples used in this publication were collected as part of NIH/NIAMS (NO1-AR-2-2265). A.M.E. receives support from the NIH National Center for Advancing Translational Sciences. G.K.S. receives support for research from the NIH (UG1 HD068258-06, HHSN272201300017I, 1UL1TR002553-01, R21AI132677) and the Centers for Disease Control and Prevention (200-2012-53663). She chairs an Independent Data Monitoring Committee for GlaxoSmithKline (RSV vaccine trials). M.C.W. receives support for research from the NIH (1R01-HD076676-01A1 and 1K24-AI143971), National Institute of Allergy and Infectious Diseases (HHSN272201500006I and HHSN272201300017I), NICHD (HHSN275201000003I), FDA (5U18-FD006298), and the industry for drug development in adults and children.

S.J. Balevic, MD, MHS, Department of Rheumatology and Immunology, and Department of Pediatrics, Duke University School of Medicine, and Duke Clinical Research Institute; C.P. Hornik, MD, PhD, Duke Clinical Research Institute, and Department of Pediatrics, Duke University School of Medicine; T.P. Green, MD, MS, Department of Pediatrics, Northwestern University, Feinberg School of Medicine; M.E. Clowse, $M D, M P H$, Department of Rheumatology and Immunology, Duke
University School of Medicine; D. Gonzalez, PharmD, PhD, Division of Pharmacotherapy and Experimental Therapeutics, UNC Eshelman School of Pharmacy, The University of North Carolina at Chapel Hill; A.R. Maharaj, PhD, Duke Clinical Research Institute; L.E. Schanberg, $M D$, Duke Clinical Research Institute, and Department of Pediatrics, Duke University School of Medicine; A.M. Eudy, PhD, Department of Rheumatology and Immunology, Duke University School of Medicine; G.K. Swamy, MD, Division of Maternal-Fetal Medicine, Department of Obstetrics \& Gynecology, Duke University School of Medicine;

B.L. Hughes, MD, MSc, Division of Maternal-Fetal Medicine, Department of Obstetrics \& Gynecology, Duke University School of Medicine; M. Cohen-Wolkowiez, MD, PhD, Duke Clinical Research Institute, and Department of Pediatrics, Duke University School of Medicine.

Address correspondence to Dr. S.J. Balevic, Department of Rheumatology and Immunology, Duke University School of Medicine, 2301 Erwin Road, CHC, T-Level, Durham, North Carolina 27710, USA. E-mail: stephen.balevic@duke.edu

Full Release Article. For details see Reprints and Permissions at jrheum. org

Accepted for publication May 14, 2019. 
were age 41 years (14) and weight $56.1 \mathrm{~kg}$ (10.5). Overall, 257 (93.1\%) were female. Mean (SD) HCQ concentrations were extracted directly from the publication.

Pediatric study. We conducted a secondary analysis of data and samples obtained from the Atherosclerosis Prevention in Pediatric Lupus Erythematosus [APPLE (ClinicalTrials.gov: NCT00065806)] trial ${ }^{9}$. We analyzed frozen serum samples (obtained between 2003-2007) for HCQ concentration using a validated high-performance liquid chromatography/ mass spectrometry assay at a commercial laboratory (NMS Labs). The assay has been previously described ${ }^{10}$. We included children from the APPLE trial who (1) provided at least 2 serum samples (baseline and 12-month study visits); and (2) were receiving HCQ at both visits. We included a total of 131 children (262 samples) in our analysis, with these average (range) baseline characteristics: age 15.6 years (10.3-21.7) and weight of $61.2 \mathrm{~kg}$ (32.9-118.9); $80.9 \%$ of participants were female. We computed the mean (SD) HCQ concentration across all patients separately for the baseline and 12-month study visits; HCQ dosing information was not available from the APPLE trial. Concentrations below the quantifiable limit $(<10 \mathrm{ng} / \mathrm{ml})$ were imputed as $5 \mathrm{ng} / \mathrm{ml}$.

Adult pregnancy study. We previously published a 1-compartment population PK model for HCQ during pregnancy and postpartum using data from 50 patients (145 serum concentrations) with rheumatic disease ${ }^{10,15}$. From this study, we extracted the mean (SD) of HCQ concentrations collectively for all doses, and separately for the 400-mg dose. Because concentrations were stratified by trimester in the original study, we reported the range of mean HCQ concentrations across all trimester/postpartum visits. Because of non-adherence to HCQ in the original study, the final PK dataset included 45 patients with 135 concentrations. Of these 135 concentrations, $22(16.3 \%)$ were from the first trimester, 51 (37.8\%) were second trimester, 37 (27.4\%) were third trimester, and 25 (18.5\%) were postpartum. Average (range) characteristics across all sampling timepoints included weight $78.3 \mathrm{~kg}(51.7-124.4)$ and serum creatinine 0.64 $\mathrm{mg} / \mathrm{dl}(0.3-1.2)$. Maternal age at conception was 31.5 years (20.4-44.4), and 31 mothers $(68.9 \%)$ were white. The most common diagnoses were SLE $(n=24)$, rheumatoid arthritis $(n=6)$, and undifferentiated connective tissue disease $(\mathrm{n}=5)$.

Dosing simulations. To provide an example of how population PK models can simulate the doses required to achieve target drug concentrations, we conducted dosing simulations using our published PK model of HCQ in pregnancy ${ }^{10}$. The model included linear scaling of weight on apparent volume of distribution and estimated between-subject variability in the apparent clearance. The population estimate [\% relative standard error (RSE)] was $18501 / 70 \mathrm{~kg}(53 \%)$ for apparent volume of distribution, and $51 \mathrm{l} / \mathrm{h}(8 \%)$ for apparent clearance. Between-subject variability was $44 \%$ for apparent clearance (\%RSE 4). With this model, we used Phoenix NLME (version 8.2; Certara) to conduct dosing simulations for pregnant patients with rheumatic disease. Using the population estimates for apparent volume of distribution, apparent clearance, the population estimates for between-subject variability in clearance, and residual error models described in the original manuscript ${ }^{10,15}$, we simulated 1000 datasets for each of 4 dosing regimens. This replicates simulated serum concentrations every $2 \mathrm{~h}$ over a 5-day period for each of 45 simulated patients using their first visit weight. We assumed a maintenance dosing of HCQ $400 \mathrm{mg}$ orally once daily for 3 months. After 3 months of maintenance therapy, the following HCQ dosage regimens were simulated: Regimen A: a loading dose of $600 \mathrm{mg}$ every 12 $\mathrm{h}$ orally on Day 1 followed by $400 \mathrm{mg}$ every $24 \mathrm{~h}$ for 4 days ${ }^{16}$; Regimen B: $200 \mathrm{mg}$ orally every $8 \mathrm{~h}$ for 10 days (dosing regimen used in clinical report by Gautret, et al ${ }^{8}$; Regimen C: $600 \mathrm{mg}$ orally every $24 \mathrm{~h}$ for 5 days; and Regimen D: $400 \mathrm{mg}$ orally every $24 \mathrm{~h}$ for 5 days (standard-of-care dosing for most patients with rheumatic diseases $)^{15}$. From a safety perspective, the range of concentrations we observed clinically during pregnancy ranged from $<0.01 \mathrm{mg} / \mathrm{l}$ to $1.1 \mathrm{mg} / \mathrm{l}$ (median $0.16-0.43$ depending on dosage and trimester $)^{15}$; therefore, we selected $1.1 \mathrm{mg} / \mathrm{l}$ as the surrogate ceiling for safety during dosing simulations.

\section{RESULTS}

Average HCQ exposure. Mean HCQ concentrations observed from the clinical studies compared to the target in vitro concentrations are outlined in Table 1 . The mean serum and plasma HCQ concentrations were below the lowest target HCQ exposure $(0.48 \mathrm{mg} / \mathrm{l})$ in all studies; however, consistent with the wide variability in HCQ pharmacokinetics, the SD included concentrations that reached or exceeded $0.48 \mathrm{mg} / \mathrm{l}$ in some cases. Assuming the highest EC50 (corresponding to a total plasma concentration of 4.1 $\mathrm{mg} / \mathrm{l}$ ), all studies had about one-tenth or less exposure than necessary for in vitro viral inhibition.

Dosing simulations. Figure 1 demonstrates the results of the dosing simulation for each dosing regimen in pregnant adults already receiving longterm HCQ. Consistent with the observed data, the simulation of pregnant adults receiving the most common dosing for rheumatic diseases (400 mg orally once a day, Regimen D), would not achieve median target exposures reported in the Gautret, et al ${ }^{8}$ and Yao, et $\mathrm{al}^{4}$ studies; however, a dosage of $600 \mathrm{mg}$ once a day (Regimen C) obtained peak median target exposure after the first dose and average target exposure after the second dose. Regimen B had the least fluctuation, but peak concentrations did not achieve target exposure until after 48 $\mathrm{h}$ of the increased dosage. From a safety perspective, the 95\% of simulated concentrations did not exceed the safety threshold (maximum $1.1 \mathrm{mg} / \mathrm{l}$ ) ${ }^{15}$ for any regimens. However, maximum simulated concentrations exceeded $1.1 \mathrm{mg} / \mathrm{l}$ for all regimens.

\section{DISCUSSION}

We compared observed HCQ drug concentrations in plasma/serum across several populations to characterize average HCQ exposure in patients with rheumatic diseases receiving longterm treatment with $\mathrm{HCQ}$. We found that most patients, including children and pregnant/non-pregnant adults, do not achieve total serum concentrations shown to inhibit SARS-CoV-2 in vitro, especially at standard dosages $<400 \mathrm{mg} /$ day. This suggests that current HCQ dosing strategies are unlikely to be effective in the setting of SARS-CoV-2 viremia, where serum concentrations most directly approximate the in vitro study. Nonetheless, owing to wide between-patient variability in HCQ PK, a minority of patients may achieve the lowest in vitro target concentrations with conventional dosing, particularly with good medication adherence. Because patients receiving longterm HCQ are already at steady state, our modeling suggests that changes to serum concentrations occur quickly with an increase in dosage. Achieving concentrations with antiviral activity quickly after viral exposure may be particularly important, because the in vitro activity of HCQ against SARS-CoV-2 may depend on accumulation into cells ${ }^{4}$.

When considering the optimal dosage of HCQ, one must remain cognizant that the therapeutic window for HCQ in

Personal non-commercial use only. The Journal of Rheumatology Copyright @ 2020 . All rights reserved. 
Table 1. Average hydroxychloroquine (HCQ) concentrations in clinical studies compared to lowest target EC50 concentration.

\begin{tabular}{|c|c|c|c|c|c|}
\hline Study & Population & Sample Size & $\begin{array}{l}\text { All Doses, HCQ } \\
(\mathrm{mg} / \mathrm{l}), \text { Mean }(\mathrm{SD})\end{array}$ & $\begin{array}{l}\text { 400-mg Dose, HCQ } \\
(\mathrm{mg} / \mathrm{l}), \text { Mean }(\mathrm{SD})\end{array}$ & $\begin{array}{l}\text { Target Plasma HCQ } \\
(\mathrm{mg} / \mathrm{l}), \text { EC50 } 0.72 \mathrm{uM}\end{array}$ \\
\hline Balevic $^{15}$ (serum) & $\begin{array}{l}\text { Pregnant adults, rheumatic diseases } \\
\text { (North America) }\end{array}$ & 50 & $\begin{array}{c}0.305-0.424 \\
(0.193-0.261)\end{array}$ & $\begin{array}{c}0.319-0.441 \\
(0.195-0.281)\end{array}$ & \\
\hline Balevic (serum) & Children, SLE (North America) & 131 & $\begin{array}{l}\text { Baseline: } 0.3(0.229) \\
\text { 12-month: } 0.287(0.211)\end{array}$ & NA & 0.48 \\
\hline $\begin{array}{l}\text { Morita }^{13} \text { (plasma) } \\
\text { Mok }^{14} \text { (serum) }\end{array}$ & $\begin{array}{l}\text { Adults, cutaneous or SLE (Japan) } \\
\text { Adults, SLE (China) }\end{array}$ & $\begin{array}{c}90 \\
276\end{array}$ & $\begin{array}{l}0.136-0.197(0.045-0.079) \\
0.202-0.437(0.152-0.281)\end{array}$ & $\begin{array}{l}0.192(0.046) \\
0.437(0.281)\end{array}$ & \\
\hline
\end{tabular}

EC50: half-maximal effective concentrations; SLE: systemic lupus erythematosus; NA: not applicable.

Regimen A

$600 \mathrm{mg}$ orally every 12 hours on Day 1 $400 \mathrm{mg}$ orally every 24 hours Days 2-5

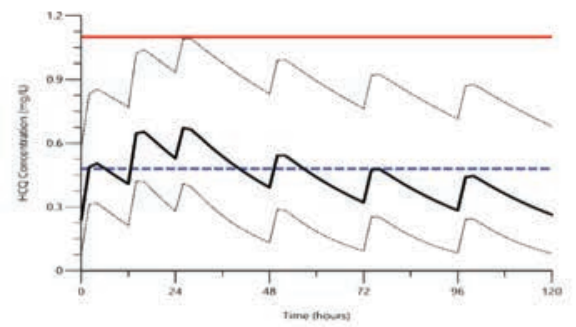

Regimen C

$600 \mathrm{mg}$ orally every 24 hours for 5 days

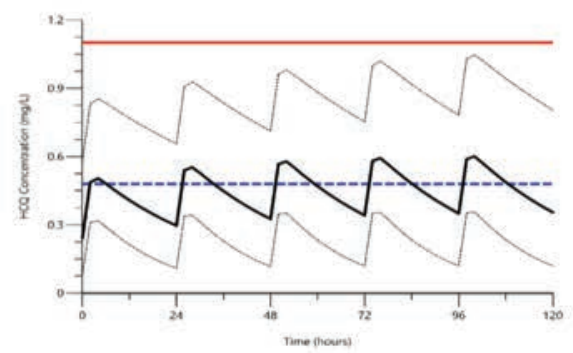

Regimen B

$200 \mathrm{mg}$ orally every 8 hours for 10 days

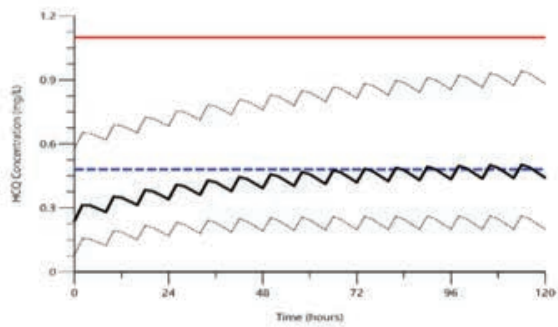

Regimen D

400 mg orally every 24 hours for 5 days (Standard of care)

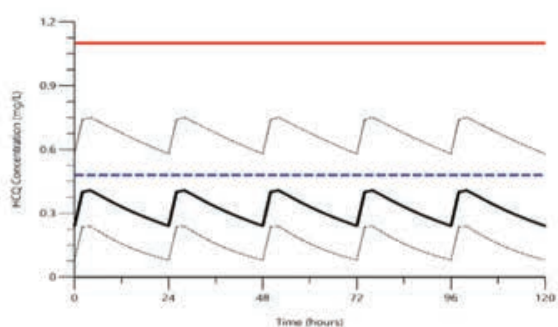

Figure 1. Dosing simulations. The solid black lines represent median predicted serum hydroxychloroquine concentration from the model (mg/l). Upper dashed black lines represent the $95 \%$ of simulated data whereas the lower dashed black line represents the $5 \%$. The dashed blue line represents the target serum concentration derived from Yao, et al ${ }^{4}(0.48 \mathrm{mg} / 1$, assuming EC50 of $0.72 \mu \mathrm{M})$. The solid red line represents the highest observed concentration in the clinical study as a surrogate ceiling for safety $(1.1 \mathrm{mg} / \mathrm{l})$. EC50: half-maximal effective concentrations.

the setting of SARS-CoV-2 is unknown. Moreover, because HCQ distributes extensively into tissues and becomes trapped in lysosomes, tissue concentrations may be significantly higher than blood concentrations ${ }^{5}$. For example, studies in albino rats suggest concentrations of HCQ in the lung are $>200-600$ times higher than plasma ${ }^{17}$. High lung concentrations highlight a potential mechanism by which HCQ may be efficacious for SARS-CoV-2, despite subtherapeutic blood concentrations; however, chronic dosing may be important because lung concentrations in animal studies accumulate over several months ${ }^{18}$. Second, it is possible that HCQ exerts an effect through antiviral, antithrombotic, and antiinflammatory properties ${ }^{18}$; these mechanisms of action may occur at different concentrations. This is particularly relevant because the reported in vitro differences in EC50 for HCQ treatment for SARS-CoV-2 vary from 0.72 to $6.14 \mu \mathrm{M}$; this higher plasma target, corresponding to a total plasma concentration of $4.1 \mathrm{mg} / \mathrm{l}$, is likely not achievable in patients without incurring substantial toxicity. Moreover, the in vitro-derived EC50 represented the concentration needed for $50 \%$ viral inhibition; concentrations of $6.7 \mathrm{mg} / \mathrm{l}$ are needed for complete viral inhibition (e.g., EC100) Additionally, the available data for most published studies did not allow us to characterize HCQ peak and trough

Personal non-commercial use only. The Journal of Rheumatology Copyright @ 2020 . All rights reserved. 
concentrations; however, average concentration is an appropriate antiviral exposure target because this most closely approximates the conditions for efficacy based on the in vitro test. Accordingly, it is crucial for future research to better characterize the mechanism of action for HCQ in the setting of SARS-CoV-2, as well as goal drug concentrations in the site of action (i.e., lung cells or interstitial fluid), before any specific dosing adjustments can be recommended for HCQ.

Population PK models are a powerful tool that relate dose and patient characteristics (e.g., weight) to drug concentrations. Once a target drug concentration is identified, models are used to conduct simulations to optimize dosing. As an example of this approach, we simulated several proposed dosage regimens being used for SARS-CoV-2 in a cohort of pregnant women already receiving HCQ for rheumatic diseases, and compared simulated results with the concentrations observed by Gautret, et al $(0.46 \mathrm{mg} / \mathrm{l} \pm 0.2)^{8}$ and predicted by Yao, et al $(0.48 \mathrm{mg} / \mathrm{l})^{4}$. Assuming these target concentrations are efficacious, a HCQ dosage of $600 \mathrm{mg}$ once daily consistently achieved median target concentrations for most pregnant women with rheumatic disease (Regimen C), whereas a dosage of $200 \mathrm{mg}$ every $8 \mathrm{~h}$ (total daily dose of $600 \mathrm{mg}$, Regimen B) achieved peak target concentrations only after $48 \mathrm{~h}$. Further, owing to a higher volume of distribution for HCQ in late pregnancy ${ }^{10}$, individual dosing needs may differ by trimester. From a safety perspective, the $95 \%$ of simulated concentrations did not exceed the safety threshold (maximum $1.1 \mathrm{mg} / \mathrm{l})^{15}$ for any regimen. However, maximum simulated concentrations exceeded $1.1 \mathrm{mg} / \mathrm{l}$ for all regimens because of high inter-individual variability.

There are some limitations to our work. First, serum/ plasma measurements for HCQ are known to have higher variability compared to whole blood ${ }^{13}$. Despite this limitation, current published studies for SARS-CoV-2 have either directly reported serum concentrations ${ }^{8}$, or used an in vitro platform that mimics free drug in plasma ${ }^{4}$. While differences between serum and whole blood are reviewed elsewhere ${ }^{10,15}$, serum may be prone to less confounding from the hematologic disturbances seen in SLE or COVID-19, potentially making it preferred over whole blood. Moreover, assuming a whole blood-to-plasma ratio between 3.8 and $7.2^{7,13}$, target whole blood concentrations (e.g., 1.79-3.38 mg/l) still exceed average concentrations observed by CostedoatChalumeau in a large cohort of patients with SLE ${ }^{19}$. HCQ concentrations may also be influenced by medication adherence, polymorphisms in drug-metabolizing enzymes, concomitant medications, and changes in renal function, among other factors ${ }^{10,12,15,18}$. For example, HCQ whole blood levels were $0.699 \mathrm{mg} / \mathrm{l}$ lower in non-adherent compared to adherent patients with SLE (corresponding to about $0.07-0.184 \mathrm{mg} / \mathrm{l}$ in plasma ${ }^{20}$. Therefore, while adherence may optimize the proportion of patients able to achieve the lowest EC50 target, the data suggest patients are unable to achieve the highest in vitro EC50 target or EC100.
Also, it is possible that prolonged sample storage (e.g., about 16 yrs for the oldest sample) introduced degradation or higher concentration variability for the pediatric study. Despite this possibility, the overall range of concentrations we observed in the pediatric study were highly consistent with adult and pediatric literature values ${ }^{21}$. For our population PK model in pregnancy, we conducted dosing simulations beyond those directly observed in our pregnancy cohort, but we do not expect any dose-related changes to the drug's PK model due to known linear drug disposition ${ }^{7}$. Additionally, we assumed the EC50 values represent the unbound drug at the target site (e.g., lung interstitial fluid) needed to mediate an antiviral effect. Notably, other modeling approaches (e.g., PBPK) can be used to directly simulate bound or unbound concentrations in target tissues such as the lung. Last, because of assumptions in our modeling ${ }^{10}$ and intrinsically high inter-individual variability in HCQ PK, there was less precision surrounding the estimate for volume of distribution. As a result of the high inter-individual variability in HCQ PK, we recommend that clinical studies measure HCQ concentrations to confirm actual participant exposure when investigating the use of HCQ in the setting of SARS-CoV-2.

Despite early enthusiasm for HCQ as a treatment for SARS-CoV-2, we caution against the universal clinical use of higher dosages of HCQ and highlight the need to conduct high-quality clinical trials to clarify effectiveness, safety, and target exposures. Recently, the US Food and Drug Administration highlighted safety concerns of HCQ, including heart rhythm abnormalities, and recommended the drug be administered in connection with COVID-19 only in a hospital or clinical trial setting ${ }^{22}$. Unpublished data from a Veterans Administration study also suggest higher mortality for HCQ in COVID-19. To this, we highlight concerns that widespread prescribing of higher dosages without supportive data may exacerbate a drug shortage for HCQ, leading to worse outcomes for other patients with rheumatic diseases ${ }^{23}$. For example, patients with SLE who are unable to access HCQ are 2.5 times more likely to have a disease flare ${ }^{24}$, leading to potentially life-threatening complications. This is particularly relevant given methodological concerns of recent clinical studies of HCQ for SARS-CoV-2, including a high dropout rate, lack of randomization, surrogate outcomes, low sample sizes, and potential effect interaction with azithromycin ${ }^{8}$. Accordingly, we emphasize the need for continued study of HCQ for SARS-CoV-2. To characterize the effect of acute versus chronic dosing, we advocate for the inclusion into trials of patients with rheumatic disease already receiving $\mathrm{HCQ}$.

We found that the average patient receiving longterm treatment with HCQ for rheumatic diseases, including children and non-pregnant/pregnant adults, are unlikely to achieve total serum or plasma concentrations shown to inhibit SARS-CoV-2 in vitro, especially at total daily dosages $<400 \mathrm{mg}$. Nonetheless, the therapeutic window for HCQ in

Personal non-commercial use only. The Journal of Rheumatology Copyright $(\subset) 2020$. All rights reserved. 
the setting of SARS-CoV-2 is unknown, and well-designed clinical trials are urgently needed to characterize the efficacy, mechanism of action, and target exposures for HCQ. Patients receiving HCQ long term may have tissue concentrations far exceeding that of serum/plasma, and accordingly, it is essential to include patients with rheumatic diseases in clinical studies of SARS-CoV-2. Once target exposures are identified, our study demonstrates that PK models can be used to optimize dosing for patients already receiving HCQ for the treatment of rheumatic diseases.

\section{ACKNOWLEDGMENT}

The authors thank Erin Campbell, MS, for manuscript review.

\section{ONLINE SUPPLEMENT}

Supplementary material accompanies the online version of this article.

\section{REFERENCES}

1. Guan WJ, Ni ZY, Hu Y, Liang WH, Ou CQ, He JX, et al. Clinical characteristics of coronavirus disease 2019 in China. N Engl J Med 2020;382:1708-20.

2. Zhu N, Zhang D, Wang W, Li X, Yang B, Song J, et al; China Novel Coronavirus Investigating and Research Team. A novel coronavirus from patients with pneumonia in China, 2019. N Engl J Med 2020;382:727-33.

3. Devaux CA, Rolain JM, Colson P, Raoult D. New insights on the antiviral effects of chloroquine against coronavirus: what to expect for COVID-19? Int J Antimicrob Agents 2020 Mar 12 (E-pub ahead of print).

4. Yao X, Ye F, Zhang M, Cui C, Huang B, Niu P, et al. In vitro antiviral activity and projection of optimized dosing design of hydroxychloroquine for the treatment of severe acute respiratory syndrome coronavirus 2 (SARS-CoV-2). Clin Infect Dis 2020 Mar 9 (E-pub ahead of print).

5. Arnold SL, Buckner F. Hydroxychloroquine for treatment of SARS$\mathrm{CoV}-2$ infection? Improving our confidence in a model-based approach to dose selection. Clin Transl Sci 2020 Apr 8 (E-pub ahead of print).

6. Costedoat-Chalumeau N, Leroux G, Piette JC, Amoura Z. Why all systemic lupus erythematosus patients should be given hydroxychloroquine treatment? Joint Bone Spine 2010;77:4-5.

7. Tett SE, Cutler DJ, Day RO, Brown KF. A dose-ranging study of the pharmacokinetics of hydroxy-chloroquine following intravenous administration to healthy volunteers. Br J Clin Pharmacol 1988;26:303-13.

8. Gautret P, Lagier JC, Parola P, Hoang VT, Meddeb L, Mailhe M, et al. Hydroxychloroquine and azithromycin as a treatment of COVID-19: results of an open-label non-randomized clinical trial. Int J Antimicrob Agents 2020 Mar 20 (E-pub ahead of print).

9. Schanberg LE, Sandborg C, Barnhart HX, Ardoin SP, Yow E, Evans GW, et al; Atherosclerosis Prevention in Pediatric Lupus Erythematosus Investigators. Use of atorvastatin in systemic lupus erythematosus in children and adolescents. Arthritis Rheum 2012;64:285-96.

10. Balevic SJ, Green TP, Clowse ME, Eudy AM, Schanberg LE, Cohen-Wolkowiez M. Pharmacokinetics of hydroxychloroquine in pregnancies with rheumatic diseases. Clin Pharmacokinet 2019;58:525-33.
11. Furst DE. Pharmacokinetics of hydroxychloroquine and chloroquine during treatment of rheumatic diseases. Lupus 1996;5 Suppl 1:S11-5.

12. Browning DJ. Pharmacology of chloroquine and hydroxychloroquine. In: Browning DJ. Hydroxychloroquine and chloroquine retinopathy. New York: Springer; 2014:35-63.

13. Morita S, Takahashi T, Yoshida Y, Yokota N. Population pharmacokinetics of hydroxychloroquine in Japanese patients with cutaneous or systemic lupus erythematosus. Ther Drug Monit 2016;38:259-67.

14. Mok CC, Penn HJ, Chan KL, Tse SM, Langman LJ, Jannetto PJ. Hydroxychloroquine serum concentrations and flares of systemic lupus erythematosus: a longitudinal cohort analysis. Arthritis Care Res 2016;68:1295-302.

15. Balevic SJ, Cohen-Wolkowiez M, Eudy AM, Green TP, Schanberg LE, Clowse ME. Hydroxychloroquine levels throughout pregnancies complicated by rheumatic disease: implications for maternal and neonatal outcomes. J Rheumatol 2019;46:57-63.

16. Centers for Disease Control and Prevention. Information for clinicians on investigational therapeutics for patients with COVID-19 [Internet. Accessed May 19, 2020.] Available from: www.cdc.gov/coronavirus/2019-ncov/hcp/therapeutic-options.html

17. Berliner RW, Earle DP, Taggart JV, Zubrod CG, Welch WJ, Conan NJ, et al. Studies on the chemotherapy of the human malarias. VI. The physiological disposition, antimalarial activity, and toxicity of several derivatives of 4-aminoquinoline. J Clin Invest 1948; 27:98-107.

18. Rainsford KD, Parke AL, Clifford-Rashotte M, Kean WF Therapy and pharmacological properties of hydroxychloroquine and chloroquine in treatment of systemic lupus erythematosus, rheumatoid arthritis and related diseases. Inflammopharmacology 2015;23:231-69.

19. Costedoat-Chalumeau N, Amoura Z, Hulot JS, Hammoud HA, Aymard G, Cacoub P, et al. Low blood concentration of hydroxychloroquine is a marker for and predictor of disease exacerbations in patients with systemic lupus erythematosus. Arthritis Rheum 2006;54:3284-90.

20. Costedoat-Chalumeau N, Houssiau F, Izmirly P, Le Guern V, Navarra S, Jolly M, et al. A prospective international study on adherence to treatment in 305 patients with flaring SLE: assessment by drug levels and by self-administered questionnaires. Clin Pharmacol 2018;103:1074-82.

21. Laaksonen AL, Koskiahde V, Juva K. Dosage of antimalarial drugs for children with juvenile rheumatoid arthritis and systemic lupus erythematosus. A clinical study with determination of serum concentrations of chloroquine and hydroxychloroquine. Scand J Rheumatol 1974;3:103-8.

22. U.S. Food \& Drug Administration. Hydroxychloroquine or chloroquine for COVID-19: drug safety communication - FDA cautions against use outside of the hospital setting or a clinical trial due to risk of heart rhythm problems. [Internet. Accessed May 19, 2020.] Available from: www.fda.gov/safety/medical-product-safetyinformation/hydroxychloroquine-or-chloroquine-covid-19-drugsafety-communication-fda-cautions-against-use.

23. American College of Rheumatology (ACR). COVID-19 clinical guidance for adult patients with rheumatic diseases. [Internet. Accessed May 19, 2020.] Available from: www.rheumatology.org/ Portals/0/Files/ACR-COVID-19-Clinical-Guidance-SummaryPatients-with-Rheumatic-Diseases.pdf

24. Canadian Hydroxychloroquine Study Group. A randomized study of the effect of withdrawing hydroxychloroquine sulfate in systemic lupus erythematosus. N Engl J Med 1991;324:150-4. 
APPENDIX 1. List of study collaborators. The following participated in this study by enrolling patients at sites or by performing study procedures at sites: Stacy Ardoin, Esi Morgan Dewitt, C. Egla Rabinovich, Janet Ellis, Kelly Mieszkalski, Janet Wootton (Duke University Medical Center, Durham, North Carolina); Peter Chira, Joyce Hsu, Tzielan Lee, Christy Sandborg, Jan Perea (Stanford University School of Medicine, Palo Alto, California); Beth Gottlieb, Patricia Irigoyen, Jennifer Luftig, Shaz Siddiqi, Zhen Ni, Marilynn Orlando, Eileen Pagano (Cohen Children's Medical Center, New Hyde Park, New York); Andrew Eichenfield, Lisa Imundo, Deborah Levy, Philip Kahn, Candido Batres, Digna Cabral (Morgan Stanley Children's Hospital of New York-Presbyterian, New York, New York); Kathleen A. Haines, Yukiko Kimura, Suzanne C. Li, Jennifer Weiss, Mary Ellen Riordan, Beena Vaidya (Hackensack University Medical Center, Hackensack, New Jersey); Emily von Scheven, Michelle Mietus-Snyder (University of California at San Francisco Medical Center, San Francisco, California); Earl Silverman, Lawrence Ng (Hospital for Sick Children, Toronto, Ontario, Canada); Suzanne Bowyer, Susan Ballinger, Thomas Klausmeier, Debra Hinchman, Andrea Hudgins (Indiana University School of Medicine, Indianapolis, Indiana); Marilynn Punaro, Shirley Henry, Shuzen Zhang (Texas Scottish Rite Hospital for Children, Dallas, Texas); Nora G. Singer, Elizabeth B. Brooks, Stacy Miner, Nancy Szabo, Lisabeth Scalzi (University Hospitals/Case Medical Center, Cleveland, Ohio); David Sherry, Libby Dorfeld, Sarajane Wilson, Jenna Tress (Children's Hospital of Philadelphia, Philadelphia, Pennsylvania); Deborah McCurdy, Tatiana Hernandez, Jyotsna Vitale (University of California Los Angeles Medical Center, Los Angeles, California); Marisa Klein-Gitelman, Angela Kress, Nicole Lowe, Falguni Patel (Children's Memorial Hospital, Chicago, Illinois); Carol Wallace, Stephanie Hamilton (Seattle Children's Hospital and Regional Medical Center, Seattle, Washington); Richard Silver, Katie Caldwell, Diane Kamen (Medical University of South Carolina, Charleston, South Carolina); Linda Wagner-Weiner, Becky Puplava, Atanas Lonchev (University of Chicago, Chicago, Illinois); Gloria Higgins, Monica Bacani (Nationwide Children's Hospital, Columbus, Ohio); Hermine Brunner, Cynthia Rutherford, Jamie Meyers-Eaton, Shannen Nelson, Alexei Grom (Cincinnati Children's Hospital Medical Center, Cincinnati, Ohio); Larry Jung, Teresa Conway, Lacey Frank, Lori Kuss (Creighton University Medical Center, Omaha, Nebraska); Jenny Soep, Hazel Senz (University of Colorado, Aurora, Colorado); Ann Reed, Thomas Mason, Jane Jaquith, Diana E. Paepke-Tollefsrud (Mayo Clinic, Rochester, Minnesota). 